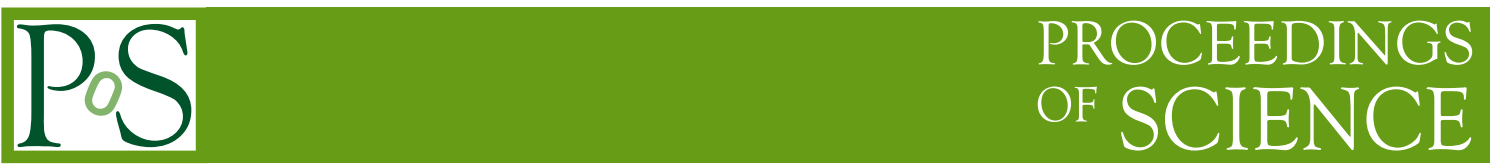

\title{
Galactic cosmic nuclei and leptons with USINE
}

\author{
Mathieu Boudaud ${ }^{* a}$, David Maurin ${ }^{b}$ \\ ${ }^{a}$ LPTHE, CNRS \& UPMC, 4 Place Jussieu, F-75252 Paris - France \\ ${ }^{b}$ LPSC, UGA \& CNRS/IN2P3, 53 avenue des Martyrs, 38026 Grenoble - France \\ E-mail: boudaudelpthe.jussieu.fr
}

USINE is a $\mathrm{C}++$ toolbox for the propagation of Galactic cosmic rays (GCRs). Starting from $\mathrm{CR}$ properties (list of nuclei and their properties, interaction cross-sections) and Galactic ingredients (source spectra, interstellar medium, radiation field, etc.), several semi-analytical propagation models are available (1D and 2D). Non-public versions of USINE were used during the last decade to determine the transport parameters, to study radioactive nuclei, and to test a possible dark matter contribution in antinuclei. This poster illustrates USINE usefulness both as a pedagogic and as a research tool. While preparing for an overdue public release, we are also implementing the recently developed 'pinching method' to have electrons and positrons in USINE.

35th International Cosmic Ray Conference - ICRC2017

10-20 July, 2017

Bexco, Busan, Korea

${ }^{*}$ Speaker. 


\section{Introduction}

The last 15 years have seen many surprises and developments on the cosmic-ray side thanks to the high statistics data provided by the PAMELA, FERMI, and AMS-02 collaborations. New features (e.g., breaks) and/or the high accuracy of these data are challenging the standard propagation models currently used in the CR community.

CRs are supposed to be accelerated by supernova remnants (and also by pulsar wind nebulae for electrons/positrons). After being released by these astrophysical systems, they are confined in the Galaxy by the magnetic field structure, diffusing on the magnetic field and interacting with the interstellar medium (ISM). A full and thorough treatment invokes magneto-hydrodynamic (MHD) calculations to encompass all the microphysics at play. However, this approach requires a precise description of the Galaxy not often accessible as well as an important CPU time to solve numerically the non-linear equations of MHD. Since the Larmor radius of CRs deflected by the turbulent magnetic field is much smaller than the Galaxy size, CR propagation can be assimilated to a random walk, and can be described by a diffusion process from the rigidity of a few GV to tens of TV. In general, numerical algorithms are required to solve the full phenomenological transport equation (e.g., GALPROP [12], DRAGON [4], or PICARD [7]), allowing to deal with any geometry and dependence for the transport parameters (diffusion, convection, reacceleration). An alternative approach is to restrict the geometry and dependences to cases for which the transport equation can be solved semi-analytically [8]. This method is faster, which is an advantage to run extensive scans over the transport parameter space.

In the following, we briefly present the semi-analytical models implemented in USINE and the code structure. For illustration purpose, we show some example plots. We finally discuss the new implementation of the pinching method allowing to deal with the propagation of cosmic ray electrons and positrons.

\section{The two-zone model and the semi-analytical method}

The Galaxy geometry is described by the two-zone models. The first zone, within which the ISM is homogeneously distributed, represents the Galactic disc of half-height $h$. The secondzone is the magnetic halo $(\mathrm{MH})$ of half-height $L$ typically lying between a few kpc and few tens of kpc. The scattering of cosmic CRs on the Galactic magnetic field is modelled by a diffusion in space with the diffusion coefficient $K(R, \vec{x})$ where $R=p / q$ is the rigidity of the cosmic ray related to its momentum $p$ and its charge $q$. Since the diffusion centres move with the Alfvén waves velocity $V_{a}$, the second-order Fermi mechanism applies and CRs could undergo a diffusive reacceleration. This process can be modelled by a diffusion in energy space with coefficient $D(E, \vec{x}) \propto V_{a}^{2} / K(R, \vec{x})$. Moreover, CRs can interact with the ISM, leading to energy losses (including ionisation and Coulomb interaction) and their destruction at rates $b$ and $\Gamma$, respectively, and radioactive species decay at rate $\tau_{\mathrm{dec}}^{-1}$. Finally, CRs undergo the effect of the Galactic wind $\vec{V}_{c}(z)$ produced by supernova explosions in the Galactic disc.

In the thin disc approximation, the density of CRs per unit of space and energy $\psi(E, t, \vec{x}) \equiv$ $d N / d^{3} x d E$ obeys the generic transport equation

$$
\partial_{t} \psi+\vec{\nabla} \cdot\left[\vec{V}_{c} \psi-K \vec{\nabla} \psi\right]+\partial_{E}\left[b \psi-2 h \delta(z) D \partial_{E} \psi\right]=Q(E, t, \vec{x})-S(E, t, \vec{x}),
$$


where $Q$ represents the injection rate of CRs in the Galaxy, whether they are primary or secondary species, whereas the sink term $S=\left(\Gamma+\tau_{\mathrm{dec}}^{-1}\right) \psi$. For more details on the method to solve 2.1, we refer the reader to $[8,11,3]$. The cosmic rays measured on the top of the Earth atmosphere have to penetrate the heliosphere where they interact with the Solar wind and the Solar magnetic field. These mechanisms can be described by a local phenomenological diffusion equation. A more straightforward approach is to use the forced-field approximation [5].

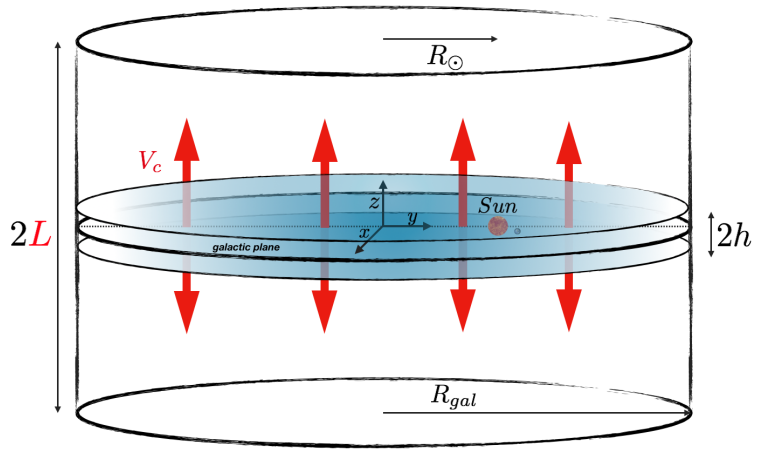

Figure 1: Galaxy geometry in the two-zone model.

\section{USINE}

The USINE code calculates the galactic flux of any CR species, solving the transport equation (2.1) in a 1D and 2D two-zone model. All input ingredients are controlled by an (USINEformatted) ASCII parameter file, including the list of propagated CRs, their parents, the ISM description, the formula for the transport and source parameters, the files for the interaction crosssections and CR data to use (USINE relies on the CRDB ${ }^{1}$ 'USINE export' format [10]), Solar modulation model and its parameters, etc. The first version of USINE was written in 1999 in C. The second version was written between 2003 and 2005 in C++ and interfaced with ROOT ${ }^{2}$. The current version (V3) is based on a complete rewriting of $\mathrm{V} 2$ in order to provide a more generic and flexible code, whose structure is illustrated in Fig.2: thanks to the many properties of C++ (abstraction, inheritance, overloading, etc.), the above classes are designed to enable generic spatial and energy dependences, using for instance pre-defined interpreted keywords for energy variables ( $R$, Etot, beta, Ekn) to be used in formulae along with any free parameters (using the Function Parser library $\left.^{3}\right)$.

Outputs of a typical USINE run are files and plots (using the CERN ROOT interface) directly comparing the calculation to CR data. As a model calculation is very fast, a text-user interface is provided for pedagogical purposes, where the user can play with the parameters, decide to compare visually the use of different cross-section files, etc. In the fit mode, USINE is interfaced with

\footnotetext{
${ }^{1}$ https://lpsc.in2p3.fr/crdb

${ }^{2}$ https://root.cern.ch

${ }^{3}$ http://warp.povusers.org/FuctionParser
} 


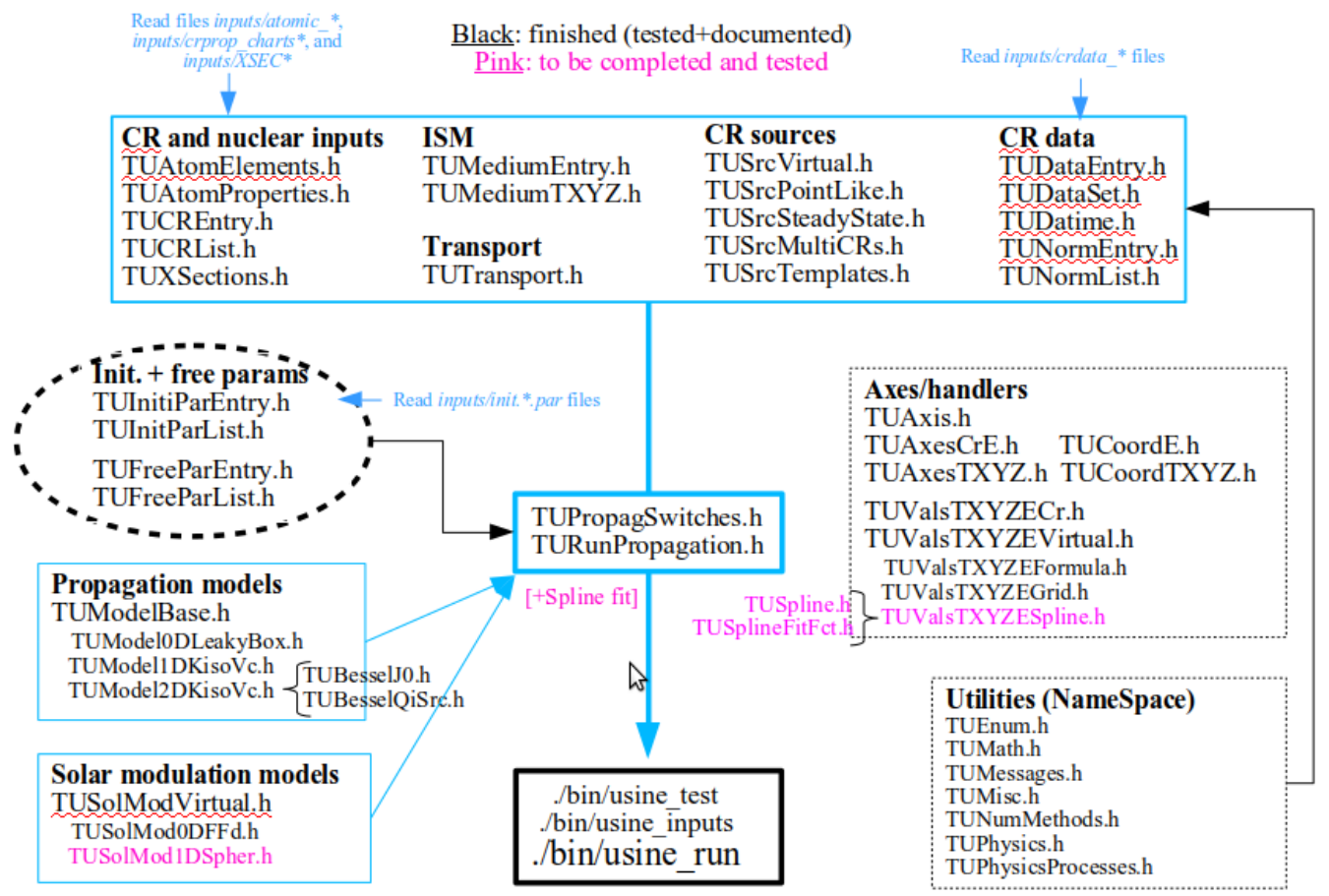

Figure 2: $\mathrm{C}++$ classes in USINE sorted according to the propagation ingredients.

MINUIT, and almost any parameters (transport, modulation level, source term) can be left free, simply by writing it in the USINE parameter file. The most recent developments of the code were dedicated to make it as user-friendly as possible. For instance, the code is now fully documented, it has extensive test functions to ensure the stability of the code as development goes, and it has been migrated on a git server in preparation of the forthcoming public release. A tutorial is also in preparation in order to walk users through the parameter files and USINE many run options.

\section{Cosmic-ray nuclei}

USINE provides a very useful tool to learn about the nature of CR nuclei and the physics behind their propagation. As an example, Fig. 3 shows the proportion of primaries (coming directly from the acceleration sites), secondaries (in one or several steps) and decay-fed (from radioactive parents) with respect to the measured flux at the Earth for each isotope (top panel) as well as each element (bottom panel), for 1,10 and $100 \mathrm{GeV} / \mathrm{n}$. USINE enables also for a given element to trace its parents (in one or several steps) and then to determine the relative part of its parents to the measured flux. This ranking is very useful since, as pointed out in [9], one of the main uncertainties for CR physics is the poor knowledge of nuclear spallation cross-sections.

Ratios of secondary to primary species (like B/C) measured at the earth are crucial to understand the $\mathrm{CR}$ propagation and to determine the propagation parameters. Nevertheless, the normalisation of the diffusion coefficient $K_{0}$ is degenerated with the size of the magnetic halo $L$. This degeneracy can be lifted with the measurement of radioactive species like ${ }^{10} \mathrm{Be}$. As a second ex- 

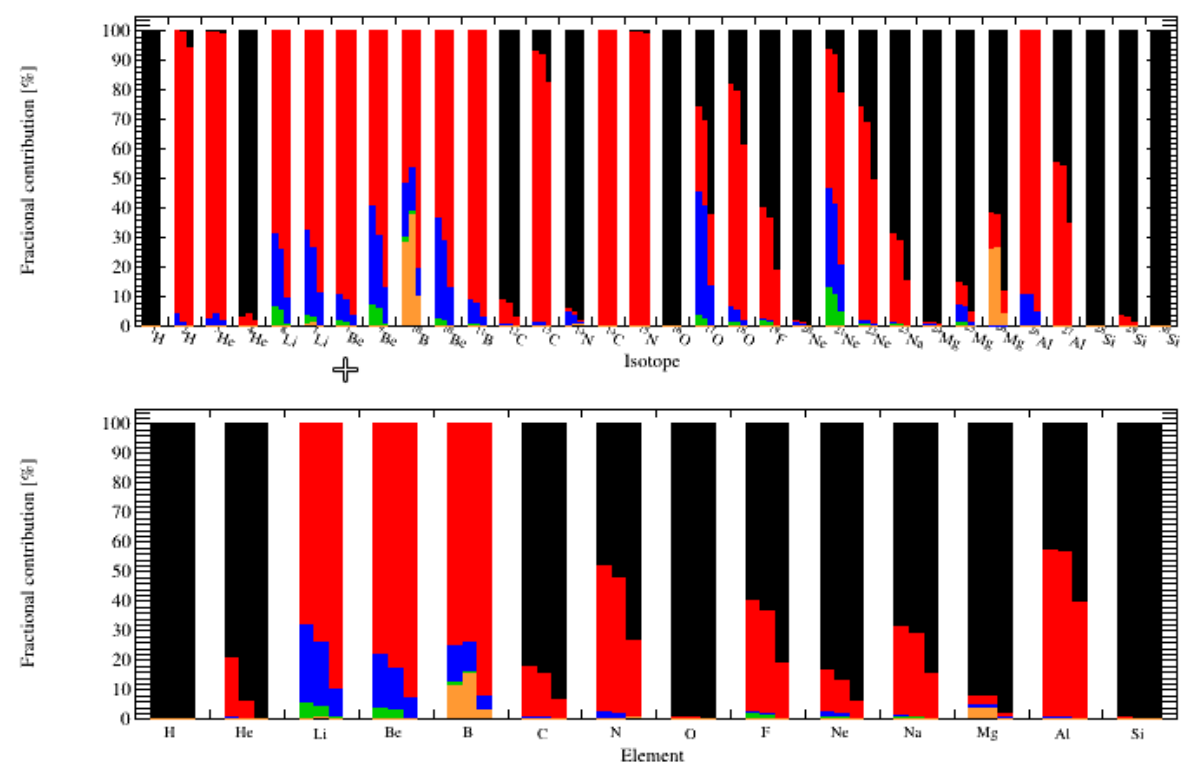

Figure 3: Fractional contribution of primaries (black), production in 1 (red), 2 (blue), or $\geq 3$ steps (green), and decay-fed contributions (orange) for 1,10 and $100 \mathrm{GeV} / \mathrm{n}$.

ample, the left panel of Fig. 4 illustrates the fractions of the parent and daughter nuclei resulting from the decay reaction ${ }^{10} \mathrm{Be} \rightarrow{ }^{10} \mathrm{~B}$ during the propagation, with respect to the case of no-decay.

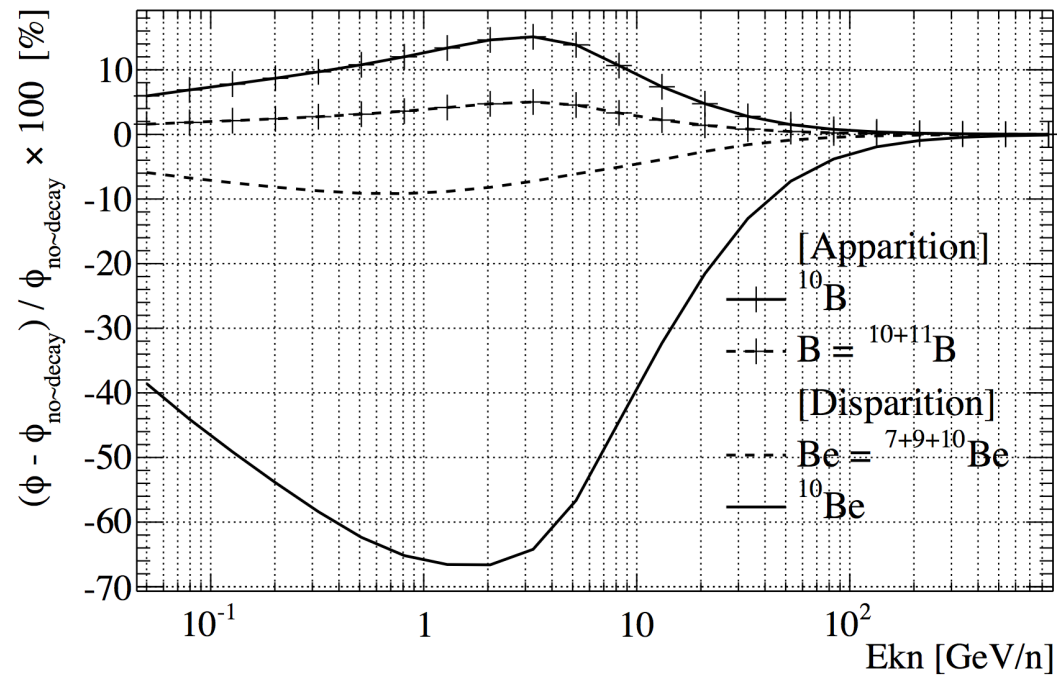

Figure 4: Relative impact of the radioactive decay ${ }^{10} \mathrm{Be} \rightarrow{ }^{10} \mathrm{~B}$ for the fluxes of ${ }^{10} \mathrm{Be}$ and ${ }^{10} \mathrm{~B}$.

The last example illustrates the fact that USINE can also be used to provide very relevant research results. Indeed, recently, the AMS-02 collaboration reported a break $\Delta \delta \simeq 0.14$ at rigidity $R_{b} \simeq 300 \mathrm{GV}$ in the measured spectra of CR proton and helium [1]. Taking advantage of the mini- 
mization module and the flexibility of the parameter settings (allowing for fully free or alternatively nuisance parameters), the hypothesis of a break in the diffusion coefficient was tested:

$$
K(R)=K_{0} \beta \frac{(R / \mathrm{GV})^{\delta}}{\left\{1+\left(R / R_{b}\right)^{\Delta \delta / s}\right\}^{s}},
$$

where $s \simeq 0.04$ is a smoothing parameter. The full analysis is reported in [6], and also presented in this conference. The analysis favours a break in the $\mathrm{B} / \mathrm{C}$ ratio, as illustrated in Fig. 5 where the data points are the AMS-02 B/C data [2].

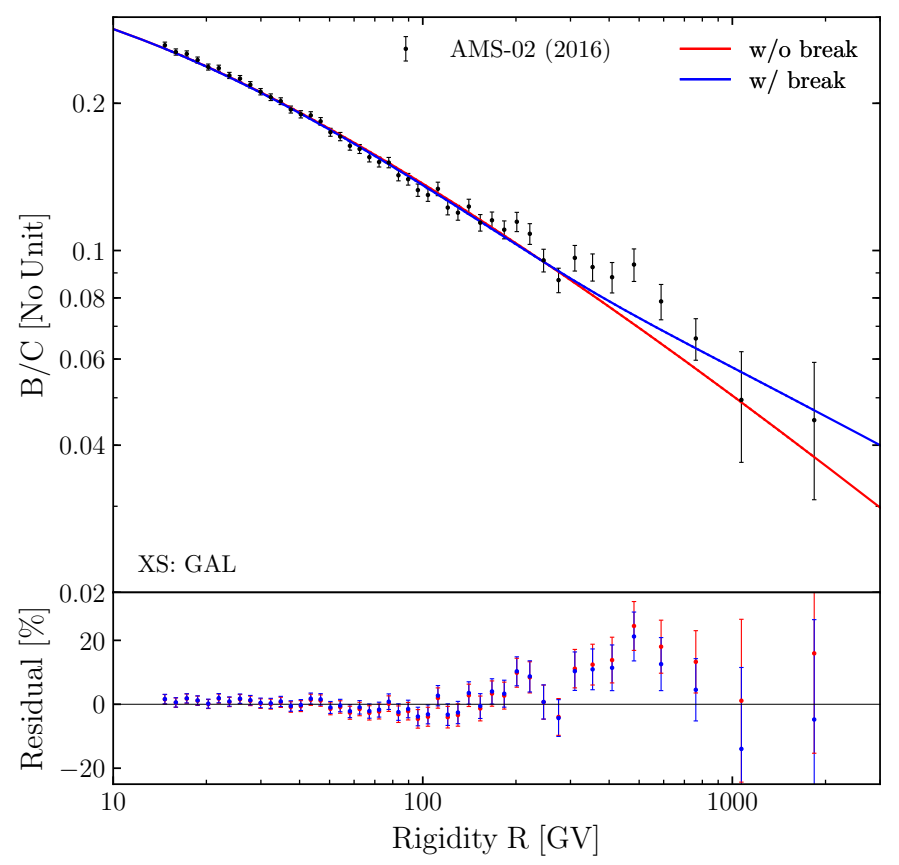

Figure 5: The predicted B/C computed with the standard diffusion coefficient (red) and with a break in the diffusion coefficient (blue) to be compared with the AMS-02 data.

\section{Cosmic ray electrons and positrons}

CR electrons and positrons undergo energy losses in the whole magnetic halo through synchrotron emission and inverse Compton scattering on the interstellar radiation field. Hence, in principle, we cannot use the same semi-analytic method as for nuclei. However, the pinching method developed in [3] allows to overcome this difficulty. As multi-species analyses in a common framework are crucial to address the difficult question of possible dark matter signals in CR data, this method is currently being added to USINE. Indeed, electrons and positrons do not have the same propagation story as for nuclei, so that they provide us with precious complementary informations, as pointed out in [3]. Fig. 6 illustrates the capability for the positron channel to reduce the parameter space of propagation. Note that the implementation for the propagation of electrons and positrons should be achieved by the end of 2018 . 


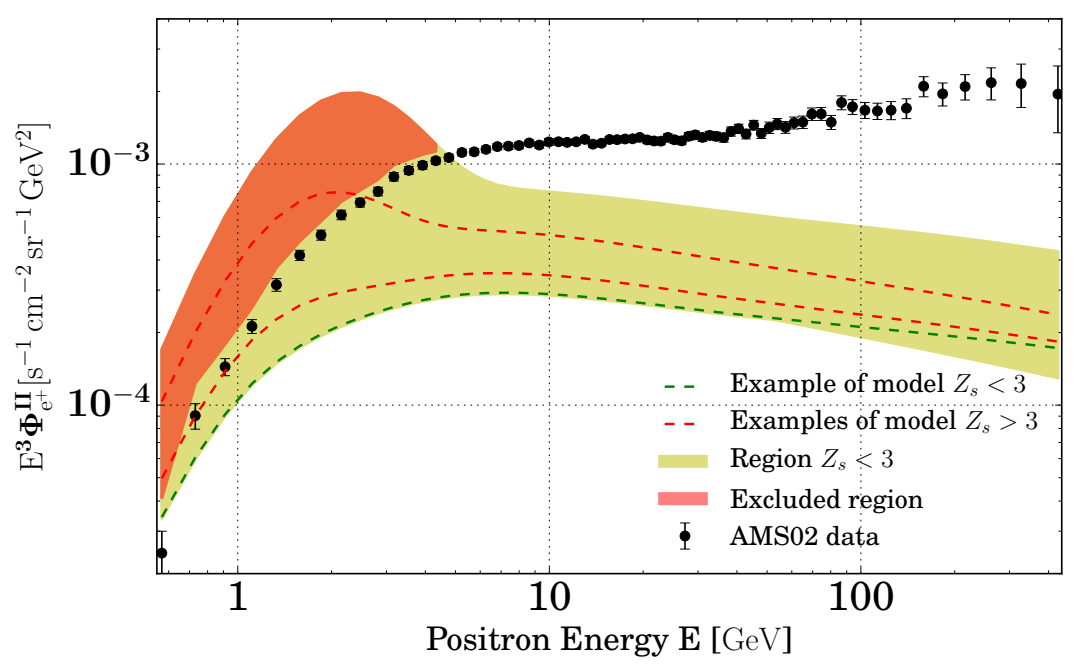

Figure 6: Complementarity between the B/C and the positron observables. The yellow band represents the predicted flux of positrons from the propagation parameters consistent with the B/C data [8]. The red band represents the region excluded at $99.7 \%$ C.L. by the AMS-02 positron data [3].

\section{Conclusion}

USINE is a C++/ROOT code dedicated to the propagation of GCR nuclei and anti-nuclei as well as electrons and positrons. Although USINE was developed for two zones (thin disc and thick halo) 1D and 2D models, it could be easily extended to more complex modelling. A lot of efforts were dedicated towards flexibility (the USINE classes for the propagation ingredients are designed to support 3D and time dependence), simplicity of the inputs (ASCII files for cross-sections, CR data, and initialisation and description of the free parameters of the study), and comparisons to data (import from CRDB, plots, and additional free parameters easily handled in the minimisation). In the context of AMS-02 data and the many young researchers entering the field, USINE should prove a fast and useful tool to learn or better understand the richness of the physics of GCRs.

\section{Aknowledgments}

MB acknowledges support from the European Research Council (ERC) under the EU Seventh Framework Program (FP7/2007-2013)/ERC Starting Grant (agreement n. 278234 NewDark project led by M. Cirelli). DM acknowledges support from the "Investissements d'avenir, Labex ENIGMASS", and by the French ANR, Project DMAstro-LHC, ANR-12-BS05-0006.

\section{References}

[1] Aguilar, M. et al., PRL 114, 171103 (2015) and PRL 115, 211101 (2015)

[2] Aguilar, M. et al., PRL 117, 231102 (2016) 
[3] Boudaud, M., Bueno, E. F., Caroff, S. et al., arXiv:1612.03924 (2016)

[4] Di Bernardo, G., Evoli, C., Gaggero, D. et al., AP 2010, 08, 006 (2010)

[5] Fisk, L. A., JGR 76, 221 (1971)

[6] Genolini Y., Serpico P.D., Boudaud M. et al., arXiv:1706.09812 (2017)

[7] Kissman, R., AP 2014, 02, 002 (2014)

[8] Maurin, D., Donato, F., Taillet, R., and Salati, P., ApJ 555, 585 (2001)

[9] Maurin, D., Putze, A., and Derome, L., A\&A 516, 67 (2010)

[10] Maurin, D., Melot, F., and Taillet, R., A\&A 569, A32 (2014)

[11] Putze, A., Derome, L., and Maurin, D., A\&A 516, 66 (2010)

[12] Strong, A.W. and Moskalenko, I.V., ApJ 509, 212 (1998) 